\title{
Exact Null Controllability, Stabilizability, and Detectability of Linear Nonautonomous Control Systems: A Quasisemigroup Approach
}

\author{
Sutrima Sutrima $\mathbb{D}^{1},{ }^{1,2}$ Christiana Rini Indrati, ${ }^{2}$ and Lina Aryati ${ }^{2}$ \\ ${ }^{1}$ Mathematics Department of Universitas Sebelas Maret, Surakarta, Indonesia \\ ${ }^{2}$ Mathematics Department of Universitas Gadjah Mada, Yogyakarta, Indonesia \\ Correspondence should be addressed to Sutrima Sutrima; zutrima@yahoo.co.id
}

Received 2 May 2018; Accepted 10 October 2018; Published 1 November 2018

Academic Editor: Lucas Jodar

Copyright (c) 2018 Sutrima Sutrima et al. This is an open access article distributed under the Creative Commons Attribution License, which permits unrestricted use, distribution, and reproduction in any medium, provided the original work is properly cited.

In the theory control systems, there are many various qualitative control problems that can be considered. In our previous work, we have analyzed the approximate controllability and observability of the nonautonomous Riesz-spectral systems including the nonautonomous Sturm-Liouville systems. As a continuation of the work, we are concerned with the analysis of stability, stabilizability, detectability, exact null controllability, and complete stabilizability of linear non-autonomous control systems in Banach spaces. The used analysis is a quasisemigroup approach. In this paper, the stability is identified by uniform exponential stability of the associated $C_{0}$-quasisemigroup. The results show that, in the linear nonautonomous control systems, there are equivalences among internal stability, stabizability, detectability, and input-output stability. Moreover, in the systems, exact null controllability implies complete stabilizability.

\section{Introduction}

In this paper we focus on linear nonautonomous control system

$$
\dot{x}(t)=A(t) x(t)+B(t) u(t), \quad x(0)=x_{0}, t \geq 0
$$

where $x(t) \in X$ is the state, $u(t) \in U$ is the control, and $X$ and $U$ are complex Hilbert spaces of the state and control, respectively; $A(t)$ is a densely defined operator in $X$ with domain $\mathscr{D}(A(t))=\mathscr{D}$, independent of $t$; and $B(t): U \longrightarrow X$ is a bounded operator such that $B(\cdot) \in$ $L_{\infty}\left(\mathbb{R}^{+}, \mathscr{L}_{s}(U, X)\right)$, where $\mathscr{L}_{s}(V, W)$ and $L_{\infty}(\Omega, W)$ denote the space of bounded operators from $V$ to $W$ equipped with strong operator topology and the space of bounded measurable functions from $\Omega$ to $W$ provided with essential supremum norm, respectively.

In the theory of control systems, controllability and stability are the qualitative control problems that play an important role in the systems. The theory was first introduced by Kalman et al. [1] for the finite dimensional of (autonomous) time-invariant systems. On its development, the theory can be generalized into controllability and stabilizability of the nonautonomous (time-varying) control systems see, e.g., [24] and the references therein. Idea of this problem is to find an admissible control $u(t)$ such that the corresponding solution $x(t)$ of the system has desired properties.

There are many various qualitative control problems that can be implemented to study the stabilizability. One of the most commonly applied qualitative control problems is null controllability. The system (1) is said to be null controllable if there exists an admissible control $u(t)$ which steers an arbitrary state $x_{0}$ of the system into 0 . The associated stabilizability problem is to find a control $u(t)=F(t) x(t)$ such that the zero solution of the closed-loop system

$$
\dot{x}(t)=[A(t)+B(t) F(t)] x(t), \quad t \geq 0,
$$

is asymptotically stable in the Lyapunov sense. In this context, the system is said stabilizable and $u(t)=F(t) x(t)$ is called the stabilizing feedback control. The complete stabilizability is one of various types of stability that is often applied to characterize the stability of the control systems. This term was 
first introduced by Wonham [5] which relates to exponential stability of the systems. Next, based on the Lyapunov function techniques, Phat [3] investigated that the null controllability guaranteed the output feedback stabilization for the nonautonomous systems. While Jerbi [6] deal the problem of stabilizability at the origin of a homogeneous vector field of degree three.

Kalman et al. [1] and Wonham [5] have shown that in the finite-dimensional autonomous control system, if the system is null controllable in finite time then it is stabilizable. However, it does not hold for the converse. Furthermore, if the system is completely stabilizable, then it is null controllable in finite time. Investigations of controllability and stabilizability in the infinite dimensional control theory are more complicated, in particular for nonautonomous systems. For non-autonomous control systems of the finite-dimensional spaces, Ikeda et al. [7] proved that if the system is null controllable, then it is completely stabilizable. As extension of the some results of [7], Phat and Ha [4] characterized the controllability via the stabilizability and Riccati equation for the linear nonautonomous systems.

The results of the stabilizability for the finite-dimensional systems can be generalized into infinite-dimensional systems. For the autonomous systems, Phat and Kiet [8] investigated relationship between stability and exact null controllability extending the Lyapunov equation in Banach spaces. The smart characterization of generator of the perturbation semigroup for Pritchard-Salamon systems was provided by Guo et al. [9]. Rabah et al. [10] prove that exact null controllability implies complete stabilizability for neutral type linear systems in Hilbert spaces. The unbounded feedback is also investigated in the paper. For nonautonomous systems, Hinrichsen and Pritchard [11] introduced a concept of radius stability for the systems under structured nonautonomous perturbations. Indeed, this concept is an advanced investigation of the stabilizable theory. In the linear nonautonomous systems in Hilbert spaces, Niamsup and Phat [12] have proved that exact null controllability implies the complete stabilizability. $\mathrm{Fu}$ and zhang [13] had established a sufficient result of exact null controllability for a nonautonomous functional evolution system with nonlocal conditions using theory of linear evolution system and Schauder fixed point theorem.

As described in our recent work [14], a quasisemigroup is an alternative approach that can be implemented to investigate the non-autonomous systems (1). This approach was first introduced by Leiva and Barcenas [15]. By this approach, $A(t)$ is an infinitesimal generator of a $C_{0}$-quasisemigroup on $X$. Sutrima et al. [16] and Sutrima et al. [17] investigated the advanced properties and some types of stabilities of the $\mathrm{C}_{0}$-quasisemigroups in Banach spaces, respectively. Even, the quasisemigroup approach can be applied to characterize the controllability of the non-autonomous control systems, although it is still limited to the autonomous controls [18]. However, until now there is no research which investigates the qualitative control problems of the nonautonomous control systems implementing $C_{0}$-quasisemigroup theory.

In this paper, we are concern on the exact null controllability, stability, stabilizability, complete stabilizability, detectability, and possible relationship among them. In paper, we identify the stability with the uniform exponential stability of the associated $C_{0}$-quasisemigroup. The organization of this paper is as follows. In Section 2, we provide the sufficient and necessary conditons for uniform exponential stability of $C_{0}$-quasisemigroup which is an extension of [17]. Relationships among stability, stabilizability, and detectability of the linear nonautonomous control systems are considered in Section 3. In Section 4, we discuss connection between exact null controllability and complete stabizability of the linear nonautonomous control systems.

\section{Uniform Exponentially Stability of $\mathrm{C}_{0}$-Quasisemigroups}

This section is a part of the main results. We first recall the definition of a strongly continuous quasisemigroups following $[15,18]$.

Definition 1. Let $\mathscr{L}(X)$ be the set of all bounded linear operators on a Hilbert space $X$. A two-parameter commutative family $\{R(t, s)\}_{s, t \geq 0}$ in $\mathscr{L}(X)$ is called a strongly continuous quasisemigroup, in short $C_{0}$-quasisemigroup, on $X$ if for each $r, s, t \geq 0$ and $x \in X$ :

(a) $R(t, 0)=I$, the identity operator on $X$,

(b) $R(t, s+r)=R(t+r, s) R(t, r)$,

(c) $\lim _{s \rightarrow 0^{+}}\|R(t, s) x-x\|=0$,

(d) there exists a continuous increasing function $M$ : $[0, \infty) \longrightarrow[1, \infty)$ such that

$$
\|R(t, s)\| \leq M(s) \text {. }
$$

Let $\mathscr{D}$ be the set of all $x \in X$ such that the following limits exist:

$$
\begin{gathered}
\lim _{s \longrightarrow 0^{+}} \frac{R(0, s) x-x}{s} \text { and } \lim _{s \longrightarrow 0^{+}} \frac{R(t, s) x-x}{s} \\
=\lim _{s \longrightarrow 0^{+}} \frac{R(t-s, s) x-x}{s}, \quad t>0 .
\end{gathered}
$$

For $t \geq 0$ we define an operator $A(t)$ on $\mathscr{D}$ as

$$
A(t) x=\lim _{s \longrightarrow 0^{+}} \frac{R(t, s) x-\mathrm{x}}{s} .
$$

The family $\{A(t)\}_{t \geq 0}$ is called the infinitesimal generator of the $C_{0}$-quasisemigroup $\{R(t, s)\}_{s, t \geq 0}$.

In the sequel, for simplicity we denote the quasisemigroup $\{R(t, s)\}_{s, t \geq 0}$ and family $\{A(t)\}_{t \geq 0}$ by $R(t, s)$ and $A(t)$, respectively.

In this paper, stability is meant to be uniform exponential stability which was introduced by Megan and Cuc [19] and was elaborated by Sutrima et al. [17].

Definition 2. A $C_{0}$-quasisemigroup $R(t, s)$ on Banach space $X$ is said to be uniformly exponentially stable if there exist constants $\alpha>0$ and $N \geq 1$ such that

$$
e^{\alpha s}\|R(t, s) x\| \leq N\|x\|
$$

for all $t, s \geq 0$ and $x \in X$. 
Definition 2 implies that if a $C_{0}$-quasisemigroup $R(t, s)$ is uniformly exponentially stable, then the classical solution $x(t)=R(0, t) x_{0}$ of abstract non-autonomous Cauchy problems

$$
\dot{x}(t)=A(t) x(t), \quad t \geq 0, x(0)=x_{0} \in \mathscr{D},
$$

converges to 0 exponentially as $t \longrightarrow \infty$.

The following theorem is a generalization of Theorem 2.4 of [15] that plays an important role in characterizing stabilizability of the system (1).

Theorem 3. Let $A(t)$ be an infinitesimal generator of $C_{0^{-}}$quasisemigroup $R(t, s)$ on a Banach space $X$. If $B(\cdot) \epsilon$ $L_{\infty}\left(\mathbb{R}^{+}, \mathscr{L}(X)\right)$, then there exists a uniquely $C_{0}$-quasisemigroup $R_{B}(t, s)$ with its infinitesimal generator $A(t)+B(t)$ such that

$$
\begin{aligned}
R_{B}(r, t) x= & R(r, t) x \\
& +\int_{0}^{t} R(r+s, t-s) B(r+s) R_{B}(r, s) x d s,
\end{aligned}
$$

for all $t, r, s \geq 0$ with $t \geq s$ and $x \in X$. Moreover, if $\|R(r, t)\| \leq$ $M(t)$, then

$$
\left\|R_{B}(r, t)\right\| \leq M(t) e^{\|B\| M(t) t} .
$$

Proof. We define

$$
\begin{aligned}
& R_{0}(r, t) x=R(r, t) x, \\
& R_{n}(r, t) x=\int_{0}^{t} R(r+s, t-s) B(r+s) R_{n-1}(r, s) x d s,
\end{aligned}
$$

for all $t, r, s \geq 0$ with $t \geq s, x \in X$, and $n \in \mathbb{N}$, and

$$
R_{B}(r, t)=\sum_{n=0}^{\infty} R_{n}(r, t)
$$

for all $r, t \geq 0$. Following the proof of Theorem 2.4 of [15] we obtain the assertions. In addition, we have

$$
\begin{aligned}
\lim _{s \longrightarrow 0} & \frac{R_{B}(t, s) x-x}{s}=\lim _{s \longrightarrow 0}\left[\frac{R(t, s) x-x}{s}\right. \\
& \left.+\frac{1}{s} \int_{0}^{s} R(t+w, s-w) B(t+w) R_{B}(t, w) x d w\right] \\
& =A(t) x+R(t, 0) B(t) R_{B}(t, 0) x=(A(t)+B(t)) \\
& \cdot x
\end{aligned}
$$

for all $x \in X$.

Corollary 4. Let $A(t)$ be an infinitesimal generator of $C_{0^{-}}$ quasisemigroup $R(t, s)$ on a Banach space $X$. If $B(\cdot) \epsilon$ $L_{\infty}\left(\mathbb{R}^{+}, \mathscr{L}(X)\right)$, then the $C_{0}$-quasisemigroup $R_{B}(t, s)$ with its infinitesimal generator $A(t)+B(t)$ satisfies integral equation

$$
\begin{aligned}
R_{B}(r, t) x & \\
= & R(r, t) x \\
\quad & \quad \int_{0}^{t} R_{B}(r+s, t-s) B(r+s) R(r, s) x d s,
\end{aligned}
$$

for all $t, r, s \geq 0$ with $t \geq s$ and $x \in X$.
Proof. With $R_{n}(r, t)$ defined in (10), it is easy to show that

$$
R_{n}(r, t)=\widetilde{R}_{n}(r, t)
$$

for all $t, r \geq 0$ and $n \in \mathbb{N}$, where $\widetilde{R}_{n}(r, t)$ is defined by

$$
\begin{aligned}
\widetilde{R}_{0}(r, t) & =R(r, t) \\
\widetilde{R}_{n}(r, t) x & =\int_{0}^{t} \widetilde{R}_{n-1}(r+s, t-s) B(r+s) R(r, s) x d s,
\end{aligned}
$$

for all $t, r, s \geq 0$ with $t \geq s, x \in X$, and $n \in \mathbb{N}$. In virtue of (11) and (14) we obtain

$$
\begin{aligned}
& R_{B}(r, t) x=R(r, t) x+\sum_{n=1}^{\infty} \widetilde{R}_{n}(r, t) x \\
&= R(r, t) x \\
&+\sum_{n=1}^{\infty} \int_{0}^{t} R_{n-1}(r+s, t-s) B(r+s) R(r, s) x d s \\
&= R(r, t) x \\
&+\int_{0}^{t} \sum_{n=1}^{\infty} R_{n-1}(r+s, t-s) B(r+s) R(r, s) x d s \\
&= R(r, t) x \\
&+\int_{0}^{t} R_{B}(r+s, t-s) B(r+s) R(r, s) x d s,
\end{aligned}
$$

for all $t, r, s \geq 0$ with $t \geq s$ and $x \in X$.

The following example illustrates the existence of the quasisemigroup $R_{B}(t, s)$.

Example 5. Let $X$ be the space of all bounded continuous real function on $[0, \infty)$ with the supremum norm. The $C_{0}$ quasisemigroup $R(t, s)$ defined by

$$
(R(t, s) x)(\xi)=x\left(\xi+s^{2}+2 s t\right), \quad \xi, t, s \geq 0,
$$

for all $x \in X$, is generated by $A(t)$, where $A(t) x(\xi)=$ $2 t(d x / d \xi)$ on domain $\mathscr{D}=\{x \in X:(d x / d \xi) \in X\}$. There exists a $C_{0}$-quasisemigroup $R_{B}(t, s)$ satisfying Theorem 3 for some operator $B$.

We define an operator $B$ by

$$
(B(t) x)(\xi)= \begin{cases}-2 t x(\xi), & 0 \leq \xi+t<1 \\ -x(\xi), & \xi+t \geq 1\end{cases}
$$

for all $x \in \mathscr{D}$. We see that $B(\cdot) \in L_{\infty}\left(\mathbb{R}^{+}, \mathscr{L}(X)\right)$ and the quasisemigroup $R_{B}(t, s)$ defined by

$$
\begin{aligned}
& \left(R_{B}(t, s) x\right)(\xi) \\
& = \begin{cases}e^{-\left(s^{2}+2 s t\right)} x\left(\xi+s^{2}+2 s t\right), & 0 \leq \xi+s^{2}+2 s t<1 \\
e^{-s} x\left(\xi+s^{2}+2 s t\right), & \xi+s^{2}+2 s t \geq 1,\end{cases}
\end{aligned}
$$

is a $C_{0}$-quasisemigroup with the infinitesimal generator $A(t)+B(t)$. 
The following theorem is an alternative version for sufficient and necessary conditions of uniform exponential stability that was given by Sutrima et al. [17].

Theorem 6. Let $R(t, s)$ be a $C_{0}$-quasisemigroup on a Banach space $X$. The following statements are equivalent.

(a) $R(t, s)$ is uniformly exponentially stable on $X$.

(b) The uniform growth bound $\omega_{0}(R)<0$.

(c) There exists $s_{0}>0$ such that $\left\|R\left(t, s_{0}\right)\right\|<1$ for all $t \geq 0$.

Proof. $(a) \Longleftrightarrow(b)$. We recall that uniform growth bound is a constant $\omega_{0}(R)$ defined by

$$
\omega_{0}(R)=\inf _{t \geq 0} \omega_{0}(t)
$$

where $\omega_{0}(t)=\inf _{s>0}((1 / s) \log \|R(t, s)\|)$.

A $C_{0}$-quasisemigroup $R(t, s)$ is uniformly exponentially stable on $X$ if and only if there exist constants $\alpha>0$ and $N \geq 1$ such that

$$
e^{\alpha s}\|R(t, s) x\| \leq N\|x\|,
$$

for all $t, s \geq 0$ and $x \in X$. This gives that

$$
\begin{aligned}
\|R(t, s)\| & \leq N e^{-\alpha s} \\
\text { or } \frac{1}{s} \log \|R(t, s)\| & \leq \frac{\log N}{s}-\alpha,
\end{aligned}
$$

for all $t, s \geq 0$. This implies that $\omega_{0}(R) \leq-\alpha<0$.

$(a) \Longrightarrow(c)$. Assume that $R(t, s)$ is uniformly exponentially stable on $X$. There exist constants $\alpha>0$ and $N \geq 1$ such that

$$
\|R(t, s)\| \leq N e^{-\alpha s}
$$

for all $t, s \geq 0$. Set $s_{0}=\log N / \alpha$, we have the assertion.

$(c) \Longrightarrow(a)$. By condition (3), there exists an increasing continuous function $M:[0, \infty) \longrightarrow[1, \infty)$ such that

$$
\|R(t, s)\| \leq M(s),
$$

for all $t, s \geq 0$. The hypothesis gives that $\omega=$ $\sup _{t \geq 0}\left\|R\left(t, s_{0}\right)\right\| \leq 1$. Let $M_{0}=\sup _{0 \leq s \leq s_{0}} M(s)$. Any $s \geq 0$ can be stated as $s=k s_{0}+r, k \in \mathbb{N} \cup\{0\}, r \in\left[0, s_{0}\right]$. Therefore, we obtain

$$
\begin{aligned}
\|R(t, s)\| & =\left\|R\left(t+k s_{0}, r\right)\right\|\left\|R\left(t, k s_{0}\right)\right\| \\
& \leq M(r)\left\|R\left(t, k s_{0}\right)\right\| \leq M_{0} \omega^{k} .
\end{aligned}
$$

If we set $\alpha=-\left(\log \omega / s_{0}\right)>0($ since $\omega \leq 1)$ and $N=M_{0} / \omega$, then

$$
\|R(t, s)\| \leq M_{0} \omega^{k}=M_{0} e^{k \log \omega} \leq N e^{-\alpha s},
$$

for all $t, s \geq 0$. This concludes that $R(t, s)$ is uniformly exponentially stable on $X$.
For a $C_{0}$-quasisemigroup $R(t, s)$ we can define a semigroup $\{E(s)\}_{s \geq 0}$ on $L_{p}\left(\mathbb{R}^{+}, X\right), 1 \leq p<\infty$, relating with $R(t, s)$. This semigroup is defined as the Howland semigroup, introduced by Chicone and Laushkin [20], which is defined by

$$
(E(s) x)(t)= \begin{cases}R(t, s) x(t-s)), & t \geq s \\ 0, & 0 \leq t<s\end{cases}
$$

for all $x \in L_{p}\left(\mathbb{R}^{+}, X\right)$.

In the sequel we use $E(s)$ to denote the semigroup $\{E(s)\}_{s \geq 0}$. It is easy to show that $E(s)$ is strongly continuous on $L_{p}\left(\mathbb{R}^{+}, X\right)$. Moreover, if $A(t)$ is the infinitesimal generator of $R(t, s)$ with domain $\mathscr{D}$, then the infinitesimal generator of $E(s)$ is given by

$$
(\Gamma x)(t)=-\frac{d x}{d t}+A(t) x(t), \quad t \geq 0,
$$

on domain

$$
\begin{aligned}
& \mathscr{D}(\Gamma)=\{x \\
& \in L_{p}\left(\mathbb{R}^{+}, X\right): x \text { is absolutely continuous, } x(t) \\
& \in \mathscr{D}\} .
\end{aligned}
$$

Theorem 7. $A C_{0}$-quasisemigroup $R(t, s)$ is uniformly exponentially stable on a Banach space $X$ if and only if the semigroup $E(s)$ is exponentially stable on $L_{p}\left(\mathbb{R}^{+}, X\right), 1 \leq p<$ $\infty$.

Proof. $(\Longrightarrow)$. Assume that $R(t, s)$ is uniformly exponentially stable on the Banach space $X$. There exist constants $N>1$ and $\alpha>0$ such that

$$
\|R(t, s) x\| \leq N e^{-\alpha s}\|x\|, \quad t, s \geq 0
$$

for all $x \in X$. For any $x \in L_{p}\left(\mathbb{R}^{+}, X\right)$ we obtain

$$
\begin{aligned}
\|E(s) x\|_{L_{p}\left(\mathbb{R}^{+}, X\right)}^{p} & =\int_{0}^{\infty}\|(E(s) x)(t)\|_{X}^{p} d t \\
& =\int_{s}^{\infty}\|R(t, s) x(t-s)\|_{X}^{p} d t \\
& \leq \int_{s}^{\infty} N e^{-\alpha p s}\|x(t-s)\|_{X}^{p} d t \\
& \leq \int_{0}^{\infty} N e^{-\alpha p s}\|x(t)\|_{X}^{p} d t \\
& =N e^{-\alpha p s}\|x\|_{L_{p}\left(\mathbb{R}^{+}, X\right)}^{p} .
\end{aligned}
$$

Hence,

$$
\|E(s) x\|_{L_{p}\left(\mathbb{R}^{+}, X\right)} \leq N e^{-\alpha s}\|x\|_{L_{p}\left(\mathbb{R}^{+}, X\right)} .
$$

This shows that $E(s)$ is exponentially stable on $L_{p}\left(\mathbb{R}^{+}, X\right)$. 
$(\Longleftarrow)$. Assume that $E(s)$ is exponentially stable on $L_{p}\left(\mathbb{R}^{+}, X\right)$. There exist constants $N>0$ and $\alpha>0$ such that

$$
\|E(s)\|_{L_{p}\left(\mathbb{R}^{+}, X\right)} \leq N e^{-\alpha s},
$$

for all $s \geq 0$. We choose $z \in X$ such that $\|z\|=1$. For any $t \geq 0$, we choose $x \in L_{p}\left(\mathbb{R}^{+}, X\right)$ such that $x\left(s_{0}\right)=z$ for some $s_{0} \in[0, t]$. Let $s_{1}=t-s_{0}$, then

$$
\begin{aligned}
\left\|R\left(t, s_{1}\right) z\right\| & =\left\|R\left(t, s_{1}\right) x\left(s_{0}\right)\right\|=\left\|R\left(t, s_{1}\right) x\left(t-s_{1}\right)\right\| \\
& \leq\left\|\left(E\left(s_{1}\right) x\right)(t)\right\| \leq N e^{-\alpha s_{1}} .
\end{aligned}
$$

By (33), there exists $s_{2} \geq 0$ such that $\left\|R\left(t, s_{2}\right)\right\|<1$, for all $t \geq 0$. So, in virtue of Theorem $6, R(t, s)$ is uniformly exponentially stable on a Banach space $X$.

The following results are Datko's version for the sufficient and necessary conditions for the uniform exponential stability of $C_{0}$-quasisemigroups which are derived from Theorem 6. These are also alternative versions of Theorem 3.7 and 3.9 of [17] with the weaker conditions.

Corollary 8. Let $1 \leq p<\infty$. A $C_{0}$-quasisemigroup $R(t, s)$ is uniformly exponentially stable on a Banach space $X$ if and only if for every $x \in X$ there exists a constant $M_{x}$ such that

$$
\int_{s}^{\infty}\|R(t, t-s) x\|^{p} d t \leq M_{x}
$$

for all $s \geq 0$ (uniformly in s).

Proof. ( $\Longrightarrow)$. Assume that $C_{0}$-quasisemigroup $R(t, s)$ is uniformly exponentially stable on a Banach space $X$. There exist constants $\alpha>0$ and $N \geq 1$ such that

$$
\|R(t, s) x\| \leq N e^{-\alpha s}\|x\| \leq N\|x\|,
$$

for all $t, s \geq 0$ and $x \in X$. Therefore, for $p \geq 1$ we have

$$
\|R(t+s, t) x\|^{p} \leq N^{p}\|x\|^{p} .
$$

This states that for every $x \in X$, a function $t \longmapsto\|R(t+s, t) x\|$ is bounded on $[0, \infty)$. Hence, the function is integrable on the interval and its value depends on $x$. So, we can choose an $M_{x}>0$ such that

$$
\int_{0}^{\infty}\|R(t+s, t) x\| d t \leq M_{x} .
$$

Using transformation of variable, the last inequality is equivalent to (35).

$(\Longleftarrow)$. Assume that for every $x \in X$ there exists an $M_{x}$ such that (35) is fulfilled. For every $s \geq 0$, it is defined a linear operator $S_{s}: X \longrightarrow L_{p}\left(\mathbb{R}^{+}, X\right)$ by $\left(S_{s} x\right)(t):=R(t+s, t) x$. By (35), every operator $S_{s}$ is closed, so every $S_{s}$ is bounded. By (35) and Uniform Boundedness Theorem, we obtain $N:=$ $\sup _{s \geq 0}\left\|S_{s}\right\|<\infty$. Therefore, for every $x \in X$ we have

$$
\begin{aligned}
& \int_{s}^{\infty}\|R(t, t-s) x\|^{p} d t=\int_{0}^{\infty}\|R(t+s, t) x\|^{p} d t \\
& \quad=\left\|S_{s} x\right\|_{L_{p}}^{p} \leq N^{p}\|x\|^{p} .
\end{aligned}
$$

This implies the exponential stability of semigroup $E(s)$ on $L_{p}\left(\mathbb{R}^{+}, X\right)$. In fact,

$$
\begin{aligned}
\int_{0}^{\infty} & \|E(s) x\|_{L_{p}\left(\mathbb{R}^{+}, X\right)}^{p} d s \\
& =\int_{0}^{\infty} \int_{s}^{\infty}\|R(t, s) x(t-s)\|_{X}^{p} d t d s \\
& =\int_{0}^{\infty} \int_{0}^{\infty}\|R(t+s, s) x(t)\|_{X}^{p} d t d s \\
& =\int_{0}^{\infty}\left(\int_{0}^{\infty}\|R(s+t, s) x(t)\|_{X}^{p} d s\right) d t \\
& \leq \int_{0}^{\infty} N^{p}\|x(t)\|_{X}^{p} d t=N^{p}\|x\|_{L_{p}\left(\mathbb{R}^{+}, X\right)}^{p} .
\end{aligned}
$$

This shows that for every $x \in L_{p}\left(\mathbb{R}^{+}, X\right)$ there exists an $M_{x}>$ 0 such that

$$
\int_{0}^{\infty}\|E(s) x\|_{L_{p}\left(\mathbb{R}^{+}, X\right)}^{p} d s \leq M_{x} .
$$

Thus, according to Lemma 5.1.2 of [21], the semigroup $E(s)$ is exponentially stable on $L_{p}\left(\mathbb{R}^{+}, X\right)$. Finally, Theorem 7 concludes that $R(t, s)$ is uniformly exponentially stable on $X$.

Corollary 9. A $C_{0}$-quasisemigroup $R(t, s)$ is uniformly exponentially stable on a Banach space $X$ if and only if for every $x \in X$ there exist constants $K>0$ and $p \geq 1$ such that

$$
\int_{0}^{\infty}\|R(t, s) x\|^{p} d s \leq K\|x\|^{p}
$$

for all $t \geq 0$ (uniformly in $t$ ).

Proof. ( $\Longrightarrow$ ). If a $C_{0}$-quasisemigroup $R(t, s)$ is uniformly exponentially stable on a Banach space $X$ and constants $\alpha>0$ and $N \geq 1$ satisfy Definition 2, then

$$
\|R(t, s) x\|^{p} \leq N^{p} e^{-\alpha p s}\|x\|^{p},
$$

for all $t, s \geq 0$ and $x \in X$. This implies that for every $x \in X$, a function $s \longmapsto\|R(t, s) x\|$ is bounded on $[0, \infty)$. Hence, the function is integrable on the interval and

$$
\int_{0}^{\infty}\|R(t, s) x\|^{p} d s \leq \frac{N^{p}}{\alpha p}\|x\|^{p}=K\|x\|^{p}
$$

for all $t \geq 0, x \in X$, and $p \geq 1$ where $K=N^{p} / \alpha p$.

$(\Longleftarrow)$. Assume that the $C_{0}$-quasisemigroup $R(t, s)$ satisfies condition (42). We set $s \geq 1$ and $m=\int_{0}^{1}\left(d w / M(w)^{p}\right)$ 
where $M$ is a function satisfying (3). Since $R(t, s)$ is a quasisemigroup, then for $x \in X$ we have

$$
\begin{aligned}
m\|R(t, s) x\|^{p} & =\int_{s-1}^{s} \frac{\|R(t, s) x\|^{p}}{M(s-r)^{p}} d r \\
& \leq \int_{0}^{s} \frac{\|R(t, s) x\|^{p}}{M(s-r)^{p}} d r \\
& \leq \int_{0}^{s} \frac{\|R(t+r, s-r)\|^{p}\|R(t, r) x\|^{p}}{M(s-r)^{p}} d r \\
& \leq \int_{0}^{s}\|R(t, r) x\|^{p} d r \leq K\|x\|^{p} .
\end{aligned}
$$

Consequently,

$$
\|R(t, s)\| \leq N_{1},
$$

for all $t, s \geq 0$, where $N_{1}=$ maks $\left\{M(1),(K / m)^{1 / p}\right\}$. If we set $s_{0}=2^{p} N_{1}^{p} K$, then

$$
\begin{aligned}
s_{0} \| R\left(t, s_{0} x \|^{p}\right. & =\int_{0}^{s_{0}}\left\|R\left(t, s_{0}\right) x\right\|^{p} d s \\
& \leq \int_{0}^{s_{0}}\left\|R\left(t+s, s_{0}-s\right)\right\|^{p}\|R(t, s) x\|^{p} d s \\
& \leq N_{1}^{p} \int_{0}^{s_{0}}\|R(t, s) x\|^{p} d s \\
& \leq N_{1}^{p} K^{p}\|x\|^{p} .
\end{aligned}
$$

Therefore,

$$
\| R\left(t, s_{0} \| \leq \frac{1}{2}<1,\right.
$$

for all $t \geq 0$. Theorem 6 concludes that $R(t, s)$ is uniformly exponentially stable on $X$.

Next, for a $C_{0}$-quasisemigroup $R(t, s)$, it is defined a convolution operator $\mathbb{G}$ to be a linear operator on $L_{p}\left(\mathbb{R}^{+}, X\right)$ by

$$
\begin{aligned}
(\mathbb{G} x)(t) & =\int_{0}^{t} R(t, s) x(t-s) d s \\
& =\int_{0}^{t} R(t, t-s) x(s) d s, \quad t \geq 0 .
\end{aligned}
$$

According to the definition of semigroup $E(s)$ in (27), the operator $\mathbb{G}$ can be written as

$$
(\mathbb{G} x)(t)=\int_{0}^{\infty}(E(s) x)(t) d s, \quad t \geq 0 .
$$

The following result shows that if $\mathbb{G}$ is bounded on $L_{p}\left(\mathbb{R}^{+}, X\right)$ and $\Gamma$ is the infinitesimal generator of $E(s)$ which is given in (28), then $-\mathbb{G}$ is the inverse of $\Gamma$.

Theorem 10. Let $E(s)$ be a $C_{0}$-semigroup defined in (27) on $L_{p}\left(\mathbb{R}^{+}, X\right)$ with its infinitesimal generator $\Gamma$ given by (28). If $u, x \in L_{p}\left(\mathbb{R}^{+}, X\right)$, then the following statements are equivalent.
(a) $u \in \mathscr{D}(\Gamma)$ dan $\Gamma u=-x$.
(b) $u=\mathbb{G} x$.

Proof. (a) $\Longrightarrow$ (b). If condition (a) holds, then by condition (d) of Theorem 2.1.10 of [21], for $s>0$ we obtain

$$
E(s) u-u=\int_{0}^{s} E(r) \Gamma u d r=-\int_{0}^{s} E(r) x d r .
$$

If we choose $t \geq 0$ such that $s>t$, then the definition of $E(s)$ gives $(E(s) u)(t)-u(t)=-u(t)$ and

$$
\begin{aligned}
\int_{0}^{s}(E(r) x)(t) d r & =\int_{0}^{t} R(t, r) x(t-r) d r \\
& =\int_{0}^{t} R(t, t-r) x(r) d r=(\mathbb{G} x)(t) .
\end{aligned}
$$

This proves statement $(\mathrm{b})$.

(b) $\Longrightarrow$ (a). Assume that the statement (b) holds. If $s>t$, then definition of $E(s)$ implies that

$$
\begin{aligned}
\int_{0}^{s}(E(r) x)(t) d r & =\int_{0}^{s} R(t, r) x(t-r) d r \\
& =\int_{0}^{t} R(t, t-v) x(v) d v=u(t) \\
& =u(t)-(E(s) u)(t) .
\end{aligned}
$$

While for $t \geq s$ we have

$$
\begin{aligned}
\int_{0}^{s} & (E(r) x)(t) d r=\int_{0}^{s} R(t, r) x(t-r) d r \\
\quad & \int_{t-s}^{t} R(t, t-v) x(v) d v \\
\quad & \int_{0}^{t} R(t, t-v) x(v) d v-\int_{0}^{t-s} R(t, t-v) x(v) d v \\
& =u(t)-R(t, s) \int_{0}^{t-s} R(t-s, t-s-v) x(v) d v \\
& =u(t)-R(t, s) u(t-s)=u(t)-(E(s) u)(t) .
\end{aligned}
$$

The both results conclude that

$$
E(s) u-u=-\int_{0}^{s} E(r) x d r, \quad s>0 .
$$

On the other hand, since

$$
E(s) u-u=\int_{0}^{s} E(r) \Gamma u d r,
$$

then this proves the statement (a).

Lemma 11. The operator $\mathbb{G}$ is bounded on $L_{p}\left(\mathbb{R}^{+}, X\right)$ if and only if $\mathbb{G} x \in L_{p}\left(\mathbb{R}^{+}, X\right)$ for every $x \in L_{p}\left(\mathbb{R}^{+}, X\right), 1 \leq p<\infty$.

Proof. By Closed Graph Theorem, it is enough to show that the mapping $x \longmapsto \mathbb{G} x$ is closed operator. 
Theorem 12. A $C_{0}$-quasisemigroup $R(t, s)$ is uniformly exponentially stable on a Banach space $X$ if and only if $\mathbb{G}$ is a bounded operator on $L_{p}\left(\mathbb{R}^{+}, X\right), 1 \leq p<\infty$.

Proof. $(\Longrightarrow)$. In virtue of Theorem 7 , if $R(t, s)$ is uniformly exponentially stable on the Banach space $X$, then the $C_{0^{-}}$ semigroup $E(s)$ is exponentially stable on $L_{p}\left(\mathbb{R}^{+}, X\right)$. There exist constants $N>1$ and $\alpha>0$ such that

$$
\|E(s) x\|_{L_{p}\left(\mathbb{R}^{+}, X\right)} \leq N e^{-\alpha s}\|x\|_{L_{p}\left(\mathbb{R}^{+}, X\right)} .
$$

By formula (50), for any $x \in L_{p}\left(\mathbb{R}^{+}, X\right)$ we have

$$
\begin{aligned}
\|\mathbb{G} x\|_{L_{p}\left(\mathbb{R}^{+}, X\right)}^{p} & =\int_{0}^{\infty}\left\|\int_{0}^{\infty}(E(s) x)(t) d s\right\|_{X}^{p} d t \\
& \leq \int_{0}^{\infty} \int_{0}^{\infty}\|(E(s) x)(t)\|_{X}^{p} d s d t \\
& \leq \frac{N}{\alpha p} \int_{0}^{\infty}\|x(t)\|_{X}^{p} d t=\frac{N}{\alpha p}\|x\|_{L_{p}\left(\mathbb{R}^{+}, X\right)}^{p} .
\end{aligned}
$$

Therefore,

$$
\|\mathbb{G} x\|_{L_{p}\left(\mathbb{R}^{+}, X\right)} \leq\left(\frac{N}{\alpha p}\right)^{1 / p}\|x\|_{L_{p}\left(\mathbb{R}^{+}, X\right)} .
$$

This states that $\mathbb{G}$ is bounded on $L_{p}\left(\mathbb{R}^{+}, X\right)$.

$(\Longleftarrow)$. If $\mathbb{G}$ is a bounded operator on $L_{p}\left(\mathbb{R}^{+}, X\right)$, then by Theorem 10 , the operator $\Gamma$ on $L_{p}\left(\mathbb{R}^{+}, X\right)$ is injective and $\Gamma u=-x$ is equivalent to $u=\mathbb{G} x$. So, $\Gamma$ is invertible, where $\Gamma^{-1}=-\mathbb{G}$. Consequently, by Theorem 3.10 of Ch.IV of [22] we obtain $s(\Gamma)<0$, where $s(\Gamma)$ denotes the spectral bound of $\Gamma$. By Theorem 1.10 of Ch.V of [22], the $C_{0}$-semigroup $E(s)$ is exponential stable on $L_{p}\left(\mathbb{R}^{+}, X\right)$. Finally, Theorem 7 gives that $R(t, s)$ is uniformly exponentially stable on $X$.

We recall that a $C_{0}$-quasisemigroup $R(t, s)$ on a Banach space $X$ is said to be exponentially bounded if there are $\omega \in \mathbb{R}$ and a function $N_{\omega}: \mathbb{R}^{+} \longrightarrow[1, \infty)$ such that

$$
e^{\omega s}\|R(t, s)\| \leq N_{\omega}(t)
$$

for all $t, s \geq 0$. In particular, from Theorems 10 and 12 for $R(t, s)$ is exponentially bounded on the Banach space $X$, we have the following corollary.

Corollary 13. Let $R(t, s)$ is an exponentially bounded $C_{0^{-}}$ quasisemigroup on a Banach space $X$ and $\Gamma$ is the infinitesimal generator of $C_{0}$-semigroup $E(s)$ on $L_{p}\left(\mathbb{R}^{+}, X\right)$. The following statements are equivalent.
(a) $R(t, s)$ is uniformly exponentially stable on $X$.
(b) $\Gamma$ is invertible, with $\Gamma^{-1}=-\mathbb{G}$.
(c) $s(\Gamma)<0$.

\section{Stabilizability and Detectability of Linear Non-Autonomous Control Systems}

Let $X, U$, and $Y$ be complex Banach spaces. Assume $B(t)$ : $U \longrightarrow X$ and $C(t): X \longrightarrow Y$ are bounded operators such that $B(\cdot) \in L_{\infty}\left(\mathbb{R}^{+}, \mathscr{L}_{s}(U, X)\right)$ and $C(\cdot) \in L_{\infty}\left(\mathbb{R}^{+}, \mathscr{L}_{s}(X, Y)\right)$. We mainly concern on the linear non-autonomous control systems on $X$ with state $x$, input $u$, and output $y$ :

$$
\begin{aligned}
& \dot{x}(t)=A(t) x(t)+B(t) u(t), \quad t \geq 0, x(0)=x_{0}, \\
& y(t)=C(t) x(t),
\end{aligned}
$$

where $x$ is an unknown function from interval $[0, \infty)$ into $X$ and $A(t)$ is the infinitesimal generator of a $C_{0}$ quasisemigroup on $X$ with domain $\mathscr{D}(A(t))=\mathscr{D}$, independent of $t$ and dense in $X$. Here, $X, U$, and $Y$ are called the state space, the control space, and the output space, respectively.

In the sequel, we assume that $p$ is a real number such that $1 \leq p<\infty$.

Definition 14. Assume that the linear non-autonomous control system (61) holds for all initial state $x_{0} \in X$ and for all input $u \in L_{p}\left(\mathbb{R}^{+}, U\right)$. The state

$$
x(t)=R(0, t) x_{0}+\int_{0}^{t} R(s, t-s) B(s) u(s) d s,
$$

$$
0 \leq t<\infty \text {, }
$$

is defined to be a mild solution of (61).

Let $R(t, s)$ be a $C_{0}$-quasisemigroup with infinitesimal generator $A(t)$. We define an input-output mapping for the non-autonomous system (61)-(62) to be an operator $\mathbb{L}$ : $L_{p}\left(\mathbb{R}^{+}, U\right) \longrightarrow L_{p}\left(\mathbb{R}^{+}, Y\right)$ by

$$
(\mathbb{L} u)(t)=C(t) \int_{0}^{t} R(t, t-s) B(s) u(s) d s, \quad t \geq 0,
$$

for all $u \in L_{p}\left(\mathbb{R}^{+}, U\right)$.

Definition 15. The linear non-autonomous control system (61)-(62) is said to be:

(a) internally stable if the $A(t)$ is the infinitesimal generator of a uniformly exponentially stable $C_{0}$ quasisemigroup $R(t, s)$;

(b) input-output stable if the corresponding input-output mapping $\mathbb{L}$ of (64) is bounded.

Definition 16. The linear non-autonomous control system (61)-(62) is said to be:

(a) stabilizable if there exists a feedback operator $F \in$ $\mathrm{L}_{\infty}\left(\mathbb{R}^{+}, \mathscr{L}_{s}(X, U)\right)$ such that $A(t)+B(t) F(t)$ is the infinitesimal generator of a uniformly exponentially stable $C_{0}$-quasisemigroup $R_{B F}(t, s)$;

(b) detectable if there exists an operator $K \in$ $L_{\infty}\left(\mathbb{R}^{+}, \mathscr{L}_{s}(Y, X)\right)$ such that $A(t)+K(t) C(t)$ is the infinitesimal generator of a uniformly exponentially stable $C_{0}$-quassemigroup $R_{K C}(t, s)$. 
In virtue of Theorem 3, if the quasisemigroup $R_{B F}(t, s)$ and $R_{K C}(t, s)$ in Definition 16 exist, then each of them satisfies the integral equation

$$
\begin{aligned}
& R_{B F}(r, t) x=R(r, t) x+\int_{0}^{t} R(r+s, t-s) B(r+s) \\
& \cdot F(r+s) R_{B F}(r, s) x d s,
\end{aligned}
$$

and

$$
\begin{aligned}
& R_{K C}(r, t) x=R(r, t) x+\int_{0}^{t} R(r+s, t-s) K(r+s) \\
& \cdot C(r+s) R_{K C}(r, s) x d s,
\end{aligned}
$$

for all $t, r, s \geq 0$ with $t \geq s$ and $x \in X$, respectively.

In the sequel, if $B$ and $C$ are operators satisfying the system (61)-(62) and the operators $F$ and $K$ in Definition 16 exist, we define the multiply operator $\mathscr{B}: L_{p}\left(\mathbb{R}^{+}, U\right) \longrightarrow$ $L_{p}\left(\mathbb{R}^{+}, X\right), \mathscr{C} \quad: \quad L_{p}\left(\mathbb{R}^{+}, X\right) \quad \longrightarrow \quad L_{p}\left(\mathbb{R}^{+}, Y\right), \mathscr{F} \quad:$ $L_{p}\left(\mathbb{R}^{+}, X\right) \quad \longrightarrow \quad L_{p}\left(\mathbb{R}^{+}, U\right)$, and $\mathscr{K}: L_{p}\left(\mathbb{R}^{+}, Y\right) \longrightarrow$ $L_{p}\left(\mathbb{R}^{+}, X\right)$ with multiplier $B, C, F$, and $K$ by

$$
\begin{aligned}
(\mathscr{B} u)(t) & =B(t) u(t), \\
(\mathscr{C} x)(t) & =C(t) x(t), \\
(\mathscr{F} x)(t) & =F(t) x(t), \\
\text { and }(\mathscr{K} y)(t) & =K(t) y(t),
\end{aligned}
$$

respectively. It is easy to show that the operators $\mathscr{B}, \mathscr{C}, \mathscr{F}$, and $\mathscr{K}$ are linear.

Theorem 17. Let $R(t, s)$ be an exponentially bounded $C_{0^{-}}$ quasisemigroup on a Banach space $X$ with its infinitesimal generator $A(t)$. The following statements are equivalent.

(a) The system (61)-(62) is internally stable.

(b) The system (61)-(62) is stabilizable and $\mathbb{G} \mathscr{B}$ is a bounded operator from $L_{p}\left(\mathbb{R}^{+}, U\right)$ to $L_{p}\left(\mathbb{R}^{+}, X\right)$.

(c) The system (61)-(62) is detectable and and $\mathscr{C G}$ is a bounded operator from $L_{p}\left(\mathbb{R}^{+}, X\right)$ to $L_{p}\left(\mathbb{R}^{+}, Y\right)$.

(d) The system (61)-(62) is stabilizable, detectable, and input-output stable.

Proof. (a) $\Longrightarrow$ (b), (c), dan (d). By Theorem 12, the operator $\mathbb{G}$ is bounded. Since $\mathscr{B}$ and $\mathscr{C}$ are bounded operators, then operator $\mathbb{L}$ is bounded. Hence, the uniform exponential stability of $R(t, s)$ and the boundedness of $B(\cdot), C(\cdot), F(\cdot)$, and $K(\cdot)$, guarantee the existence of $C_{0}$-quasisemigroup $R_{B F}(t, s)$ and $R_{K C}(t, s)$ satisfying the integral equation (65) and (66), respectively.

(b) $\Longrightarrow$ (a). By hypothesis, there exists a uniformly exponentially stable $C_{0}$-quasisemigroup $R_{B F}(t, s)$ satisfying (65) for some $F \in L_{\infty}\left(\mathbb{R}^{+}, \mathscr{L}_{s}(X, U)\right)$. In this context, $A(t)+$ $B(t) F(t)$ is the infinitesimal generator of $R_{B F}(t, s)$. Next, we define an operator $\mathbb{G}_{B F}$, where

$$
\mathbb{G}_{B F} x(t):=\int_{0}^{t} R_{B F}(t, s) x(s) d s .
$$

According to Theorem $12, \mathbb{G}_{B F}$ is a bounded operator on $L_{p}\left(\mathbb{R}^{+}, X\right)$. For $x \in L_{p}\left(\mathbb{R}^{+}, X\right)$, by Fubini Theorem and condition (b) of Theorem 2.1 of [15], we obtain

$$
\begin{aligned}
(\mathbb{G} & \left.\mathscr{B} \mathscr{F} \mathbb{G}_{B F} x\right)(t) \\
= & \int_{0}^{t} \int_{0}^{s} R(t, t-s) B(s) F(s) R_{B F}(s, s-r) d r d s \\
= & \int_{0}^{t} \int_{r}^{t} R(t, t-s) B(s) F(s) R_{B F}(s, s-r) d s d r \\
= & \int_{0}^{t} \int_{r}^{t} \frac{\partial}{\partial s}\left[R(t, t-s) R_{B F}(s, s-r)\right] d s d r \\
= & \int_{0}^{t}\left[R_{B F}(t, t-r) x(r)-R(t, t-r) x(r)\right] d r \\
= & \left(\left(\mathbb{G}_{B F}-\mathbb{G}\right) x\right)(t) .
\end{aligned}
$$

This forces that

$$
\mathbb{G}=\mathbb{G}_{B F}-(\mathbb{G} \mathscr{B}) \mathscr{F} \mathbb{G}_{B F}
$$

The boundedness of operators $\mathbb{G} \mathscr{B}, \mathscr{F}$, dan $\mathbb{G}_{B F}$ implies that the operator $\mathbb{G}$ is bounded on $L_{p}\left(\mathbb{R}^{+}, X\right)$. Finally, Theorem 12 gives that $C_{0}$-quasisemigroup $R(t, s)$ is uniformly exponentially stable on $X$. This proves the assertion.

(c) $\Longrightarrow$ (a). Detectability of the system guarantees the existence of a uniformly exponentially stable $C_{0}$-quasisemigroup $R_{K C}(t, s)$ satisfying (66) for some $K \in L_{\infty}\left(\mathbb{R}^{+}, \mathscr{L}_{s}(Y, X)\right)$. The quasisemigroup $R_{K C}(t, s)$ is generated by $A(t)+K(t) C(t)$. Analogously with previous part, we define an operator $\mathbb{G}_{K C}$, where

$$
\mathbb{G}_{K C} x(t):=\int_{0}^{t} R_{K C}(t, s) x(s) d s .
$$

The similar reason for operators (68) dan (70), the operator $\mathbb{G}_{K C}$ and $\mathbb{G}=\mathbb{G}_{K C}-\mathbb{G}_{K C} \mathscr{K}(\mathscr{C} \mathbb{G})$ are bounded on $L_{p}\left(\mathbb{R}^{+}, X\right)$. Therefore, the $C_{0}$-quasisemigroup $R(t, s)$ is uniformly exponentially stable on $X$, that is the system (61)-(62) is internally stable.

$(d) \Longrightarrow$ (a). By detectability of the system, there exists a uniformly exponentially stable $C_{0}$-quasisemigroup $R_{K C}(t, s)$ and a bounded operator $\mathbb{G}_{K C}$. Analogously with calculation (70) we have

$$
\mathbb{G} \mathscr{B}=\mathbb{G}_{K C} \mathscr{B}-\mathbb{G}_{K C} \mathscr{K} \mathscr{C} \mathscr{G} \mathscr{B} .
$$

Since $\mathbb{L}=\mathscr{C} \mathbb{G} \mathscr{B}, \mathscr{K}$, and $\mathbb{G}_{K C}$ are bounded, then operator $\mathbb{G} \mathscr{B}$ is bounded. The boundedness of $\mathscr{B}$ implies that operator $\mathbb{G}$ is bounded on $L_{p}\left(\mathbb{R}^{+}, X\right)$. Thus, $C_{0}$-quasisemigroup $R(t, s)$ is uniformly exponentially stable on $X$. In other word, the system (61)-(62) is internally stable.

We end this section with a simple example to illustrate some results for stabilizability.

Example 18. Let $X$ be the space of all bounded continuous real functions on $[0, \infty)$ with the supremum norm and 
$U$ be the space of $L_{2}(0, \infty)$. Consider the the linear nonautonomous control system

$$
\dot{x}(t)=A(t) x(t)+B(t) u(t), \quad x(0)=x_{0}, t \geq 0,
$$

on the Banach space $X$, where $A(t) x(\xi)=2 t(d x / d \xi)$ with domain $\mathscr{D}=\left\{x \in X: d x / d \xi \in X, x(0)=x_{0}\right\}, u \in U$, and

$$
B(t)= \begin{cases}t^{1 / 2} I, & 0 \leq t<1 \\ -I, & t \geq 1\end{cases}
$$

with $I$ is the identity operator.

From the Example 5, $A(t)$ is the infinitesimal generator of a $C_{0}$-quasisemigroup $R(t, s)$ defined by

$$
(R(t, s) x)(\xi)=x\left(\xi+s^{2}+2 s t\right), \quad \xi, t, s \geq 0,
$$

for all $x \in X$. It is easy to show that there exists $s_{0}>0$ such that $\left\|R\left(t, s_{0}\right)\right\|<1$ for all $t \geq 0$. In virtue of Theorem 6 , the $C_{0}$-quasisemigroup $R(t, s)$ is uniformly exponentially stable on $X$. By Definition 15, the system (73) is internally stable. Moreover, Theorem 17 implies that the system (73) is stabilizable. In fact by Example 5, we have a feedback operator $F \in L_{\infty}\left(\mathbb{R}^{+}, \mathscr{L}_{s}(X, U)\right)$ where

$$
F(t)= \begin{cases}-2 t^{1 / 2} I, & 0 \leq t<1 \\ I, & t \geq 1\end{cases}
$$

such that $A(t)+B(t) F(t)$ is the infinitesimal generator of a uniformly exponentially stable $C_{0}$-quasisemigroup $R_{B F}(t, s)$ given by

$$
\begin{aligned}
& \left(R_{B F}(t, s) x\right)(\xi) \\
& = \begin{cases}e^{-\left(s^{2}+2 s t\right)} x\left(\xi+s^{2}+2 s t\right), & 0 \leq \xi+s^{2}+2 s t<1 \\
e^{-s} x\left(\xi+s^{2}+2 s t\right), & \xi+s^{2}+2 s t \geq 1,\end{cases}
\end{aligned}
$$

for all $t, s \geq 0$ and $x \in X$.

\section{Exact Null Controllability and Complete Stabilizability}

In this section we justify the connection between exact null controllability and complete stabilizability of the linear nonautonomous control systems. The exact null controllability is special case of exact controllability. A part of the results of exact controllability in the linear non-autonomous control systems can be studied in our previous works [14].

Definition 19. The linear non-autonomous control systems (61) is said to be exactly null controllable on $[0, \tau]$ if for every $x_{0} \in X$ there exists a control $u \in L_{p}([0, \tau], U)$ such that the mild solution $x(\cdot)$ of (61) corresponding to $u(\cdot)$ satisfies $x(\tau)=x_{1}$.

Lemma 20. Let $R(t, s)$ be a $C_{0}$-quasisemigroup on a Banach space $X$ with its infinitesimal generator $A(t)$. The linear nonautonomous control systems (61) is exactly null controllable on $[0, \tau]$ if and only if $\operatorname{ran} \mathscr{B}_{\tau} \supset \operatorname{ran} R(0, \tau)$.
Proof. From the Definition 19, the system (61) is exactly null controllable on $[0, \tau]$ if and only if for any $x_{0} \in X$, there exists $u \in L_{p}([0, \tau], U)$ such that $0=R(0, \tau) x_{0}+\mathscr{B}_{\tau} u$, where $\mathscr{B}_{\tau}$ is a controllability operator from $L_{p}([0, \tau] ; U)$ to $X$ defined by

$$
\mathscr{B}_{\tau} u=\int_{0}^{\tau} R(s, \tau-s) B(s) u(s) d s .
$$

Since ran $\mathscr{B}_{\tau}$ is a subset of $X$, this is equivalent to ran $\mathscr{B}_{\tau}$ ว ran $R(0, \tau)$.

The dual operator of the controllability operator in (78) is

$$
\mathscr{B}_{\tau}^{\prime} x^{\prime}=B^{\prime}(\cdot) R^{\prime}(\cdot, \tau-\cdot) x^{\prime} \text { on }[0, \tau],
$$

where $x^{\prime}$ denotes an element of the dual space $X^{\prime}$; see $[14,18]$.

Theorem 21. Let $R(t, s)$ be a $C_{0}$-quasisemigroup on a Banach space $X$ with its infinitesimal generator $A(t)$. If there exist $\gamma, \tau>$ 0 such that

$$
\begin{aligned}
\gamma\left\|\mathscr{B}_{\tau}^{\prime} x^{\prime}\right\|_{L_{q}} & \geq\left\|R^{\prime}(0, \tau) x^{\prime}\right\|, \\
\frac{1}{p}+\frac{1}{q} & =1,
\end{aligned}
$$

then the linear nonautonomous control systems (61) is exactly null controllable on $[0, \tau]$.

Proof. Theorem 3.4 of [23] and Lemma 20 imply the assertion.

The complete stabilizability is an extension of the concept of stabilizability in Definition 16.

Definition 22. The linear nonautonomous control systems (61) is said to be completely stabilizable if for every $\alpha>0$ there exists a feedback operator $F_{\alpha} \in L_{\infty}\left(\mathbb{R}^{+}, \mathscr{L}_{s}(X, U)\right)$ such that $A(t)+B(t) F_{\alpha}(t)$ is the infinitesimal generator of a uniformly exponentially stable $C_{0}$-quasisemigroup $R_{B F_{\alpha}}(t, s)$

We have a main result of this section that is the exact null controllability implies the complete stabilizability.

Theorem 23. The linear nonautonomous control systems (61) is a exactly null controllable; then the system is completely stabilizable by a bounded feedback operator F.

Proof. Assume that the system (61) is exactly null controllable on $[0, \tau]$. For every $x_{0} \in X$, there exists $u \in L_{p}([0, \tau], U)$ such that $x\left(\tau, x_{0}, u\right)=0$, where $x(t)=x\left(t, x_{0}, u\right)$ is a solution with initial value $x_{0}$ and a control $u(t)$ given by

$$
x(t)=R(0, t) x_{0}+\int_{0}^{t} R(s, t-s) B(s) u(s) d s .
$$

We define the admissible control $u_{x_{0}} \in L_{p}([0, \infty), U)$ by

$$
u_{x_{0}}(t)= \begin{cases}u(t), & t \in[0, \tau] \\ 0, & t>\tau\end{cases}
$$


and the corresponding solution with $u_{x_{0}}$ as

$$
x_{x_{0}}(t)= \begin{cases}x(t), & t \in[0, \tau] \\ 0, & t>\tau .\end{cases}
$$

In this context, we have

$$
\begin{aligned}
J\left(u_{x_{0}}\right) & =\int_{0}^{\infty}\left(\left\|x_{x_{0}}(t)\right\|^{2}+\left\|u_{x_{0}}(t)\right\|^{2}\right) d t \\
& \leq \int_{0}^{\tau}\left(\|x(t)\|^{2}+\|u(t)\|^{2}\right) d t<\infty .
\end{aligned}
$$

This states that the hypothesis of Thorem 4.3 Ch.IV of [24] holds with $Q=R=I$. Consequently, there exists an operator function $P(t)$ on $X$ such that for feedback control $\bar{u}(t)=$ $F(t) y(t)=-B^{*}(t) P(t) y(t)$ and the corresponding solution $y(t), t \geq 0$ of

$$
\begin{aligned}
\dot{y}(t) & =A(t) y(t)+B(t) \bar{u}(t) \\
& =[A(t)+B(t) F(t)] y(t), \quad t \geq 0,
\end{aligned}
$$

satisfy

$$
J(\bar{u})=\int_{0}^{\infty}\left(\|y(t)\|^{2}+\|\bar{u}(t)\|^{2}\right) d t \leq J\left(u_{x_{0}}\right)<\infty,
$$

for all $x_{0} \in X$. However, $y(t)=R_{B F}(0, t) x_{0}, t \geq 0$, where $R_{B F}(t, s)$ is a $C_{0}$-quasisemigroup with infinitesimal generator $A(t)+B(t) F(t)$. Therefore,

$$
\int_{0}^{\infty}\left\|R_{B F}(t, s) x\right\|^{2} d t \leq \int_{0}^{\infty}\|y(t)\|^{2} d t<\infty,
$$

for all $x \in X$. By Corollary 8, the quasisemigroup $R_{B F}(t, s)$ is uniformly exponentially stable on $X$. So, there exist constants $\alpha>0$ and $N \geq 1$ such that

$$
\left\|R_{B F}(t, s) x\right\| \leq N e^{-\alpha s}\|x\|,
$$

for all $t, s \geq 0$ and $x \in X$.

On the other hand, since the system (61) is exactly null controllable on $[0, \tau]$, then

$$
R(0, \tau) x_{0}+\int_{0}^{\tau} R(s, \tau-s) B(s) u(s) d s=0 .
$$

It is given any $\alpha>0$. By multiplying the both sides of (89) by $e^{\alpha \tau}$ we have

$$
S(0, \tau) x_{0}+\int_{0}^{\tau} S(s, \tau-s) B(s) \widetilde{u}(s) d s=0,
$$

where $S(t, s)=e^{\alpha s} R(t, s)$ and $\widetilde{u}(s)=e^{\alpha s} u(s)$. In this context, $S(t, s)$ is a $C_{0}$-quasisemigroup with infinitesimal generator $A(t)+\alpha I$. This concludes that system

$$
\dot{x}(t)=[A(t)+\alpha I] x(t)+B(t) u(t), \quad \alpha>0,
$$

is exactly null controllable on $X$.

The result in (88) implies that, for any $\alpha>0$, there exist constants $\delta_{\alpha}>0, N_{\alpha}>1$ and an operator $F_{\alpha} \in$ $L_{\infty}\left(\mathbb{R}^{+}, \mathscr{L}_{s}(U, X)\right)$ such that

$$
\left\|R_{B F_{\alpha}}(t, s)\right\| \leq N_{\delta_{\alpha}} e^{-\left(\delta_{\alpha}+\alpha\right) s}=N_{\alpha} e^{-\alpha s}, \quad s \geq 0 .
$$

Therefore, the system (61) is completely stabilizable on $X$.
The converse of Theorem 23 is not always true. The following two examples, modified from [25], describe this situation.

Example 24. Consider the autonomous abstract Cauchy problem

$$
\dot{x}(t)=A x(t), \quad x(0)=x_{0}, t \geq 0,
$$

on Hilbert space $X=L_{2}\left(\mathbb{R}^{+}\right)$where $(A x)(\xi)=(d x / d \xi)(\xi)-$ $\xi x(\xi)$ with domain $\mathscr{D}=\left\{x \in X: d x / d \xi \in X, x(0)=x_{0}\right\}$.

The Cauchy problem (93) can be considered as a nonautonomous control system with $A(t)=A$ and the control operator $B(t)=0$. We shall verify that the system is completely stabilizable but not exactly null controllable. For $x_{0} \in X$, the solution of (93) is $x(t)=R(0, t) x_{0}$, where $R(t, s)$ is a $C_{0}$-quasisemigroup given by

$$
R(t, s) x(\xi)=e^{-s^{2} / 2-\xi s} x(\xi+s), \quad t, s, \xi \geq 0,
$$

with the infinitesimal generator $A(t)$. The simple calculation gives

$$
\|R(t, s)\| \leq \frac{e^{-s^{2}}}{2 s}, \quad t \geq 0, s>0 .
$$

Therefore, for any $\alpha>0$, we can choose $\alpha_{0} \in(0,1 / 2]$ such that

$$
\|R(t, s)\| \leq \frac{e^{-s^{2}}}{2 s} \leq \frac{e^{-\alpha s}}{2 \alpha} \leq \frac{e^{-\alpha s}}{2 \alpha_{0}}=N_{\alpha} e^{-\alpha s},
$$

where $N_{\alpha} \geq 1$. This shows that system (93) is completely stabilizable.

On other hand, by the definition of $R(t, s)$, there exists $x_{0} \in X$ such that $x(t)=R(0, t) x_{0} \neq 0$, for any $t \geq 0$. Hence, the system is not exactly null controllable.

Example 25. Consider the linear nonautonomous control system

$$
\dot{x}(t)=A(t) x(t)+B(t) u(t), \quad x(0)=x_{0}, \quad t \geq 0,
$$

on Hilbert space $X=L_{2}(0,1)$, where $A(t) x(\xi)=2 t(d x / d \xi)$ and $\mathscr{D}=\left\{x \in X: d x / d \xi \in X, x(0)=x_{0}\right\}$.

We shall show that the system is completely stabilizable and exactly null controllable. For any $x_{0} \in X$, solution of (97) is

$$
x(t)=R(0, t) x_{0}+\int_{0}^{t} R(s, t-s) B(s) u(s) d s,
$$

where $R(t, s)$ is a $C_{0}$-quasisemigroup defined by

$$
\begin{aligned}
& R(t, s) x(\xi) \\
& \quad= \begin{cases}x\left(\xi+s^{2}+2 s t\right), & 0 \leq \xi+s^{2}+2 s t \leq 1 \\
0, & \xi+s^{2}+2 s t>1\end{cases}
\end{aligned}
$$


with the infinitesimal generator $A(t)$. There exists $s_{0}>0$ such that $\left\|R\left(t, s_{0}\right)\right\|<1$ for all $t \geq 0$. Consequently, by Theorem 6 there exist constants $\alpha_{0}>0$ and $N \geq 1$ such that

$$
\|R(t, s)\| \leq N e^{-\alpha_{0} s}, \quad t, s \geq 0 .
$$

It is given $\alpha>0$. If $\alpha_{0}>\alpha,\left(\alpha_{0}=\alpha+h, h>0\right)$, then

$$
\|R(t, s)\| \leq N e^{-\alpha_{0} s} \leq N e^{-\alpha s}, \quad t, s \geq 0 .
$$

If $\alpha>\alpha_{0}$, we can write $\alpha=k \alpha_{0}-h$, for some $h>0$ and $k \in \mathbb{N}$. Hence,

$$
\|R(t, k s)\| \leq N e^{-k \alpha_{0} s} \leq N e^{-(\alpha+h) s} \leq N e^{-\alpha s}, \quad t, s \geq 0 .
$$

These imply that system (97) is completely stabilizable. Moreover, for any $x_{0} \in X$ we have $x(t)=R(0, t) x_{0}=0$ for $t>1$. Thus, for any control operator $B(t)$, system (97) is exactly null controllable on $[0, \tau]$ when $\tau>1$ and control $u=0$.

\section{Conclusions}

We have established the sophistication of $C_{0}$-quasisemigroups to characterize some qualitative control problems of linear nonautonomous control systems in Banach spaces including the stability, stabilizability, detectability, exact null controllability, and complete stabilizability. There are equivalences of internal stability, stabizability, detectability, and input-output stability. Also, in linear nonautonomous control systems, the exact null controllability implies complete stabilizability. Some of the obtained results are extensions of existing results in the references to infinite-dimensional and autonomous control systems.

\section{Data Availability}

The data used to support the findings of this study are available from the corresponding author upon request.

\section{Conflicts of Interest}

The authors declare that there are no conflicts of interest regarding the publication of this article.

\section{Acknowledgments}

This research is funded by Doctoral Dissertation Research Grant 2018 of Universitas Sebelas Maret Surakarta. The authors are grateful to Research Institution and Community Service of Universitas Sebelas Maret Surakarta for funding.

\section{References}

[1] R. E. Kalman, Y. C. Ho, and K. S. Narendra, "Controllability of Linear Dynamical Systems," Differrential Equations, vol. 1, pp. 189-213, 1963.

[2] V. N. Phat, "Weak asymptotic stabilizability of discrete-time systems given by set-valued operators," Journal of Mathematical Analysis and Applications, vol. 202, no. 2, pp. 363-378, 1996.
[3] V. N. Phat, "Global stabilization for linear continuous timevarying systems," Applied Mathematics and Computation, vol. 175, no. 2, pp. 1730-1743, 2006.

[4] V. N. Phat and Q. P. Ha, "New characterization of controllability via stabilizability and Riccati equation for LTV systems," IMA Journal of Mathematical Control and Information, vol. 25, no. 4, pp. 419-429, 2008.

[5] W. M. Wonham, "On Pole Assignment in Multi-Input Controllable Linear Systems," IEEE Transactions on Automatic Control, vol. AC-12, no. 6, pp. 660-665, 1967.

[6] H. Jerbi, "Asymptotic stabilizability of three-dimensional homogeneous polynomial systems of degree three," Applied Mathematics Letters, vol. 17, no. 3, pp. 357-366, 2004.

[7] M. Ikeda, H. Maeda, and S. Kodama, "Stabilization of linear systems," SIAM Journal on Control and Optimization, vol. 10, pp. 716-729, 1972.

[8] V. N. Phat and T. T. Kiet, "On the Lyapunov equation in Banach Spaces and Applications to Control Problems," vol. 29, pp. 155$166,2002$.

[9] F. Guo, Q. Zhang, and F. Huang, "Well-posedness and admissible stabilizability for Pritchard-Salamon systems," Applied Mathematics Letters, vol. 16, no. 1, pp. 65-70, 2003.

[10] R. Rabah, G. Sklyar, and P. Barkhayev, "Exact null controllability, complete stabilizability and continuous final observability of neutral type systems," International Journal of Applied Mathematics and Computer Science, vol. 27, no. 3, pp. 489-499, 2017.

[11] D. Hinrichsen and A. J. Pritchard, "Robust stability of linear evolution operators on Banach spaces," SIAM Journal on Control and Optimization, vol. 32, no. 6, pp. 1503-1541, 1994.

[12] P. Niamsup and V. N. Phat, "Linear time-varying systems in Hilbert spaces: exact controllability implies complete stabilizability," Thai Journal of Mathematics, vol. 7, no. 1, pp. 189-200, 2009.

[13] X. Fu and Y. Zhang, "Exact null controllability of nonautonomous functional evolution systems with nonlocal conditions," Acta Mathematica Scientia, vol. 33, no. 3, pp. 747-757, 2013.

[14] Sutrima, Ch. R. Indrati, and L. Aryati, "Controllability and Observability of Nonautonomous Riesz-Spectral Systems," Abstract and Applied Analysis, vol. 2018, Article ID 4210135, 10 pages, 2018.

[15] H. Leiva and D. Barcenas, "Quasi-semigroups, Evolution Equation and Controllability," Notas de Matematicas, vol. 109, pp. 129, 1991.

[16] Sutrima, Ch. R. Indrati, L. Aryati, and Mardiyana, "The fundamental properties of quasi-semigroups," Journal of Physics: Conference Series, vol. 855, no. 1, Article ID 012052, 9 pages, 2017.

[17] Sutrima, C. Rini Indrati, and L. Aryati, "Stability of C0 quasi semigroups in Banach spaces," in Proceedings of the 1st Ahmad Dahlan International Conference on Mathematics and Mathematics Education, AD-INTERCOMME 2017, Indonesia, October 2017.

[18] D. Bárcenas, H. Leiva, and A. Tineo Moya, "The dual quasisemigroup and controllability of evolution equations," Journal of Mathematical Analysis and Applications, vol. 320, no. 2, pp. 691-702, 2006.

[19] M. Megan and V. Cuc, "On Exponential Stability of C0-QuasiSemigroups in Banach Spaces," Le Matematiche, vol. 54, no. 2, pp. 229-241 (2001), 1999.

[20] C. Chicone and Y. Latushkin, Evolution Semigroups in Dynamical Systems and Differential Equations, vol. 70 of Mathematical 
Surveys and Monographs, American Mathematical Society, Providence, RI, USA, 1999.

[21] R. F. Curtain and H. Zwart, An Introduction to In finiteDimensional Linear Systems Theory, Springer, 1995.

[22] K.-J. Engel and R. Nagel, One-Parameter Semigroups for Linear Evolution Equations, vol. 194 of Graduate Texts in Mathematics, Springer, 1999.

[23] R. F. Curtain and A. J. Pritchard, Infinite Dimensional Linear Systems, vol. 8 of Lecture Notes in Control and Information Sciences, Springer, Berlin, Germany, 1978.

[24] J. Zabczyk, Mathematical Control Theory: An Introduction, Birkhuser, Boston, Mass, USA, 1992.

[25] A. Randhi, Spectral Theory for Positive Semigroups and Applications, Salento University Publishing, Lece, Italy, 2002. 


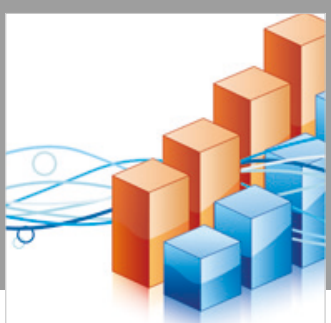

Advances in

Operations Research

\section{-n-m}
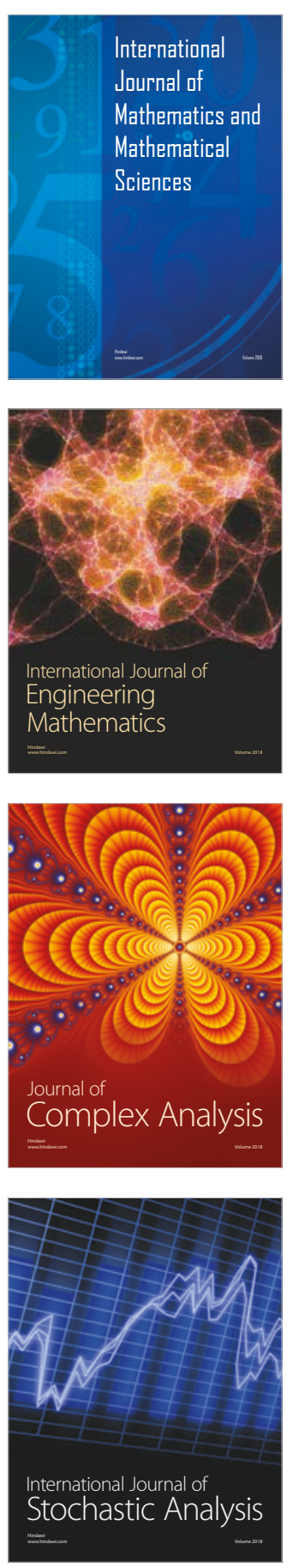
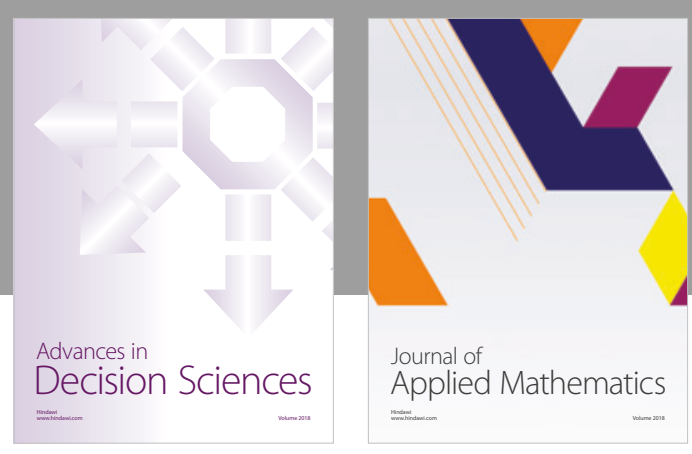

Journal of

Applied Mathematics
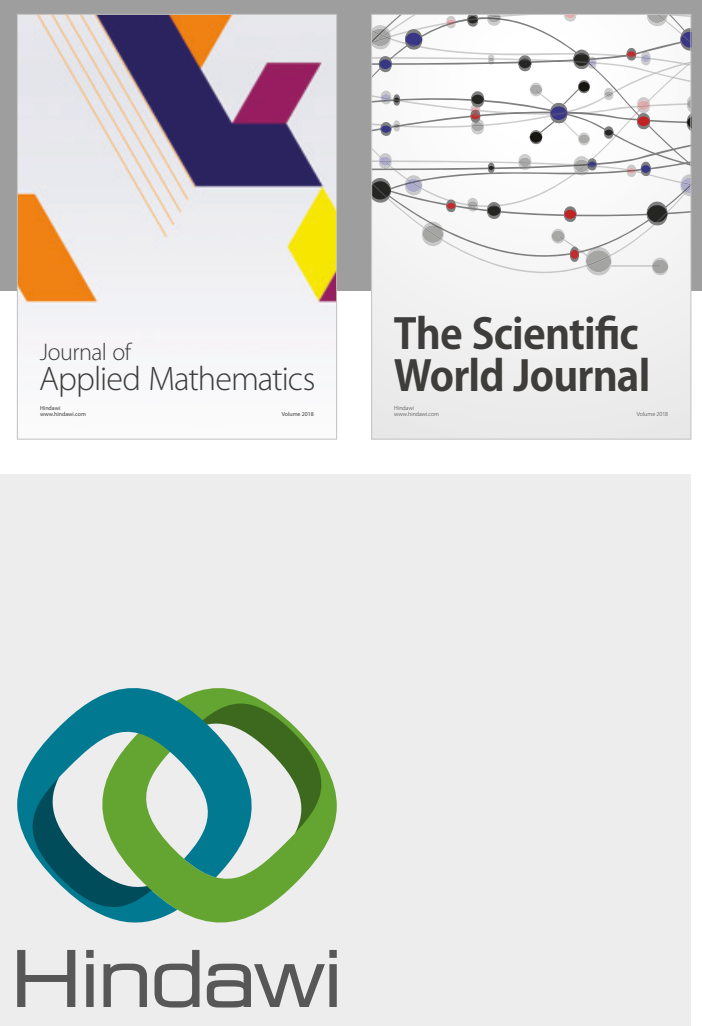

Submit your manuscripts at

www.hindawi.com

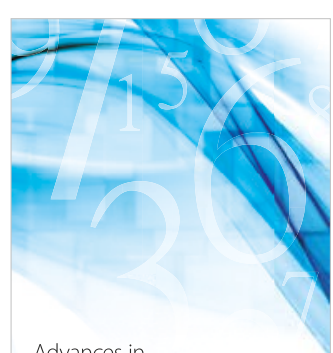

Advances in
Numerical Analysis
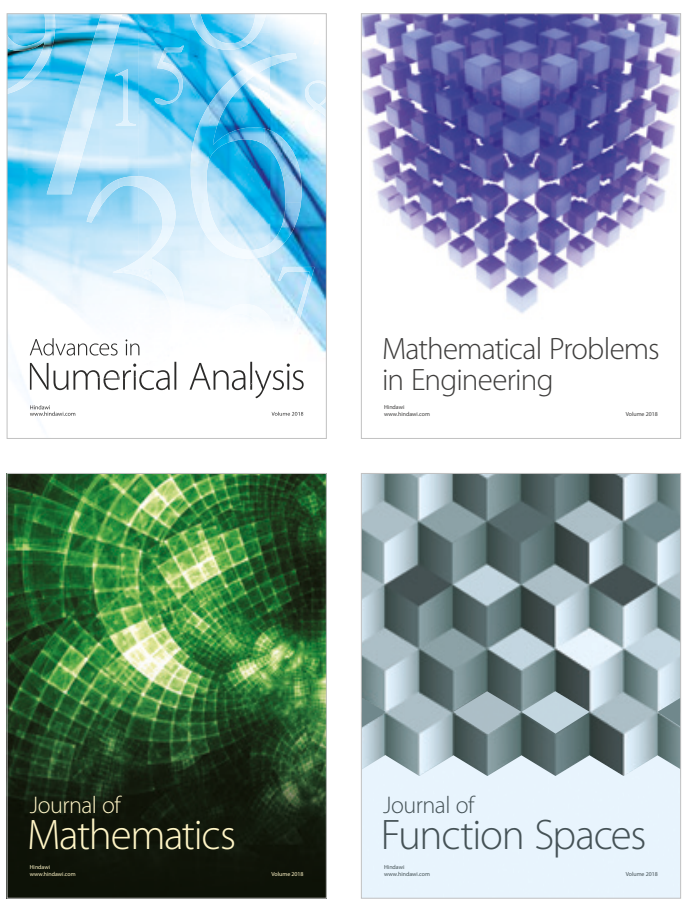

Mathematical Problems in Engineering

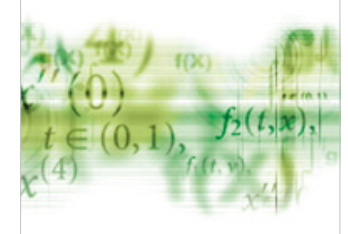

International Journal of

Differential Equations

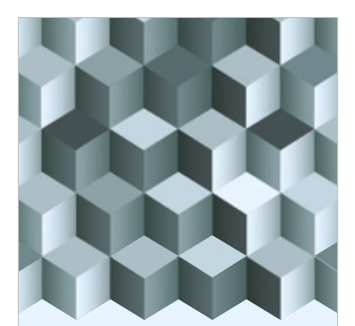

Journal of

Function Spaces

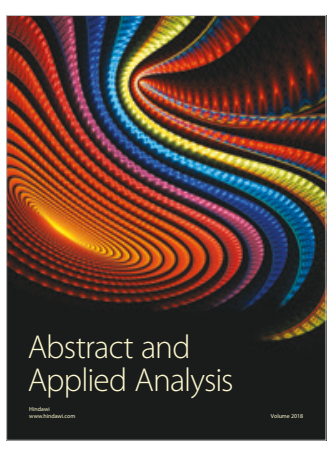

The Scientific

World Journal

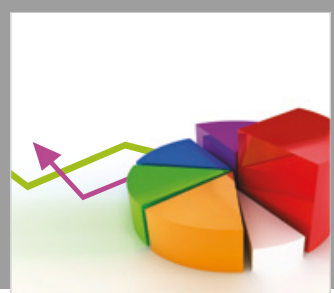

Journal of

Probability and Statistics
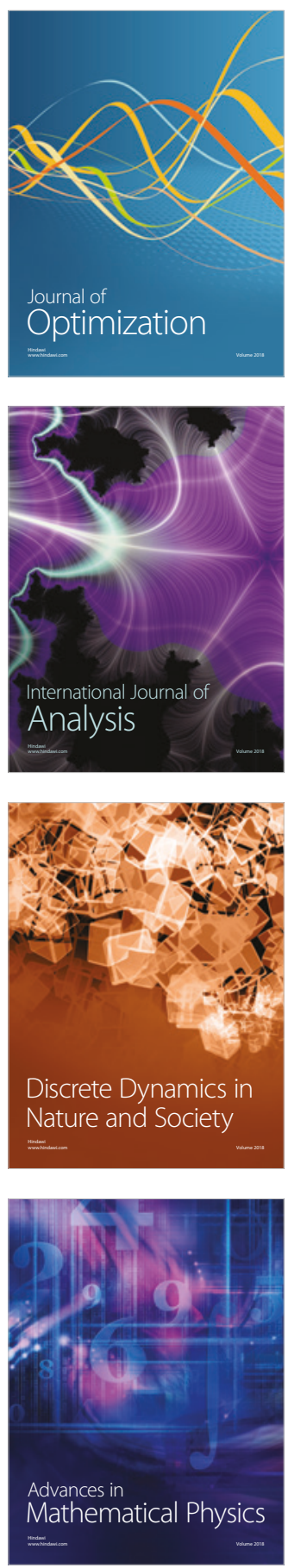